in vivo 35 : $907-911(2021)$

doi:10.21873/invivo.12330

\title{
A Finnish Version of RAND-36-Item Health Survey Versus Structured Interview 8 Years Postoperatively
}

\author{
IINA SAIMANEN $^{1 *}$, VIIVI KUOSMANEN ${ }^{1 *}$, JUKKA HARJU $^{1 *}$, TUOMAS SELANDER $^{2}$, \\ SAMULI ASPINEN ${ }^{1}$ and MATTI ESKELINEN ${ }^{1}$ \\ ${ }^{1}$ Department of Surgery, Kuopio University Hospital and School of Medicine, \\ University of Eastern Finland, Kuopio, Finland; \\ ${ }^{2}$ Science Service Center, Kuopio University Hospital, Kuopio, Finland
}

\begin{abstract}
Background/Aim: The aim was to assess the 8year health status after minicholecystectomy (MC) versus laparoscopic cholecystectomy (LC) for gallstone disease (GS) by using the RAND-36 Health Survey. Patients and Methods: Initially, 88 patients with symptomatic GS disease were randomized to undergo either $M C(n=44)$ or $L C$ (n=44). RAND-36 survey was performed 8 years postoperatively. Results: In three RAND-36 domains (social functioning, role physical, role emotional) $M C$ procedure was significantly better than LC. In MC patients, the 8-year postoperative scores of social functioning $(p<0.001)$, role physical $(p=0.002)$ and role emotional $(p<0.001)$ were significantly higher than the age-and gender-adjusted Finnish reference scores. Conclusion: The Finnish version of the RAND-36 survey can be used as a valid and reliable method for measuring the quality of life and long-term outcome of cholecystectomy patients following surgery.
\end{abstract}

The core outcome measures following surgery have classically been complications such as morbidity, mortality, requirement of analgesia, number of postoperative analgesic doses and long-term outcome including post-operative symptoms, disease recurrence and long-term complications (1-3). However, several patient-rated outcome measures, such as Beck Depression Inventory (BDI) (4), Montgomery-

This article is freely accessible online.

*These Authors contributed equally to this study.

Correspondence to: Matti Eskelinen, MD, $\mathrm{PhD}$, School of Medicine, University of Eastern Finland, P.O. Box 100, FI-70029 KYS, Finland. Tel: +358 17173311, Fax: +358 17172611, GSM: +358 400969444, e-mail: matti.eskelinen@kuh.fi

Key Words: RAND-36, telephone interview, quality of life, postoperative, cholecystectomy.
Åsberg Depression Rating Scale (5), Spielberger State-Trate Anxiety Inventory (STAI) (6), Numeric Rating Scale (NRS) (7) and Brief Pain Inventory (BPI) (8) have been reported as important patient-rated indicators for evaluating treatment results and quality of life (QoL) after surgery.

One of the most frequently used health-related QoL tools for assessing post-surgical outcomes is the Short Form Health Survey Questionnaire (SF-36) (9-12). The RAND-36 instrument contains the same questions as SF-36, but minor differences exist in scoring general health $(\mathrm{GH})$ and bodily pain (BP) scales. The Finnish version of the RAND-36-Item Health Survey has been validated and provides reference values for Finnish population (9). We have previously reported short-term health related QoL after minicholecystectomy (MC) versus laparoscopic cholecystectomy (LC) using RAND-36 $(10,11)$. However, the long-term post-cholecystectomy outcome using RAND-36 has not been addressed in MC versus LC patients. Thus, we designed the present study to i) assess long-term health status using RAND-36 in MC versus LC, ii) compare long-term post-cholecystectomy RAND-36 values with reference values in the general Finnish population. Moreover, we performed a telephone-interview to further assess QoL at 8 years after MC versus LC.

\section{Patients and Methods}

The study was approved by the Ethics Committee and registered in the ClinicalTrials.gov database (ClinicalTrials.gov Identifier: NCT01723540, Figure 1), and design and surgical techniques used in the study are presented in our earlier paper (12) and Figure 1. The operations were carried out between September 2010 and April 2012 (12).

A telephone interview was conducted between January 2019 and January 2020. A structured questionnaire including assessment of residual symptoms, de novo symptoms, QoL and cosmesis was completed. Moreover, health related QoL was assessed postoperatively using the validated Finnish version of the RAND36 by mail prior the interview (9). The eight health domains were calculated from the 36 questions as instructed by the RAND-36 


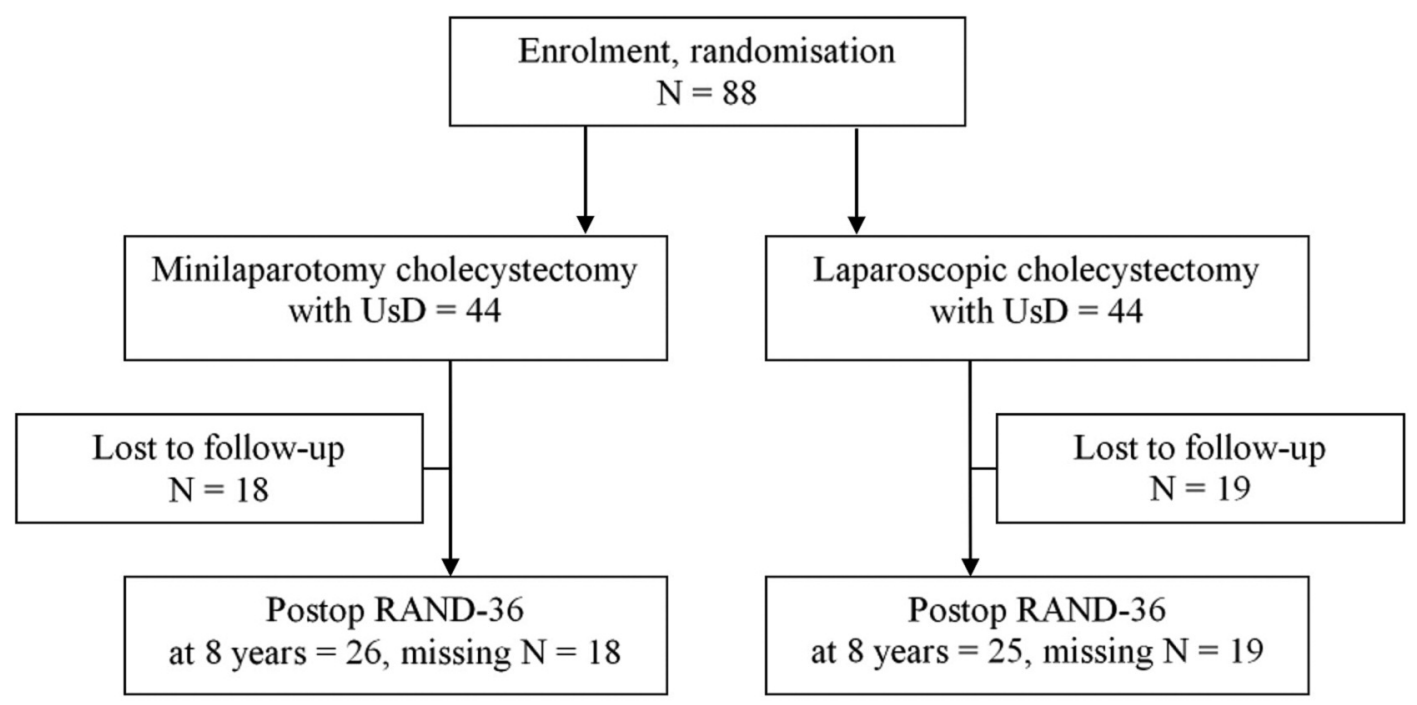

Figure 1. The flowchart. MC: Minicholecystectomy; LC: laparoscopic cholecystectomy; UsD: ultrasonic knife dissection.

item health survey (9). The design of our structured telephone interview performed in this study is presented in our recent paper (11) and in Table I. The data were analyzed with a statistical software program (IBM SPSS Statistics 26.0, IBM, Armonk, NY, USA). Differences in the baseline characteristics between groups were tested by Fisher's exact test and in the case of continuous data the analysis was performed by independent samples $t$-test. Group differences at four time points were tested by the Mann-Whitney $U$ test and the Wilcoxon signed rank test.

\section{Results}

Thirty-eight $(38 / 44=86.4 \%)$ in the MC group and thirty-five $(35 / 44=79.5 \%)$ patients in the LC group were reached for the structured questionnaire phone interview by phone at 8 years following surgery. Further on, $26(26 / 44,59.1 \%)$ of the MC patients and $25(25 / 44,56.8 \%)$ of the LC patients returned the Finnish version of RAND-36 questionnaire 8-year following surgery. The consort figure and clinical data of the study are shown in Figure 1 and in Table II.

At the structured interview no statistically significant differences between MC and LC patients were shown; residual abdominal symptoms $(p=0.61)$, right upper quadrant pain $(p=0.89)$, reflux symptoms $(p=0.16)$, diarrhea/loose stools $(p=0.14)$, avoid fatty/fried food $(p=0.90)$, overall satisfaction with the procedure $(p=0.40)$, cosmetic satisfaction $(p=0.52)$, recommend the procedure to others $(p=0.12)$, quality of life $(p=0.60)$ (Table I).

The eight RAND-36 domains with scores in MC versus LC patients 8 years postoperatively are shown in Table III. In three RAND-36 domains; social functioning ( $p=0.019)$, role physical ( $p=0.043$ ), role emotional $(p=0.008)$, the scores were significantly higher in the MC than LC patients. The 8- year scores of eight RAND-36 domains in the MC and LC groups versus the Finnish reference scores are shown in Figure 2. The 8-year postoperative scores of social functioning $(p<0.001)$, role physical $(p=0.002)$ and role emotional $(p<0.001)$ were significantly higher than the ageand gender -adjusted Finnish reference scores.

\section{Discussion}

The present study applied the Finnish version of the RAND36 , which was originally developed to examine health related QoL. The validity and reference values for the Finnish population have been verified for the RAND-36. We have previously studied the short-term outcomes after cholecystectomy, but the current literature lacks long-term health related patient centered outcome data. Moreover, a comparison to a reference population was deemed important and thus RAND-36 was selected to provide a reliable outcome instrument for the task.

In our previous report (10), we found no statistically significant QoL differences when comparing the MC and LC patients except for the higher score in 'health change' subscale at 4 weeks following surgery in the MC group. In addition, when study groups combined, four RAND-36 domains showed statistically significant improvement in health related QoL four weeks following surgery. Moreover, six RAND-36 domains improved 6 months postoperatively compared to the baseline. We also presented mid-term outcomes after MC vs. LC and showed that RAND-36 scores improved in several domains in MC and LC patients with a similar postoperative course (11). The linear mixed effect model was used to test the overall significance of RAND-36 
Table I. The results of the structured telephone interview at 8 years.

\begin{tabular}{|c|c|c|c|}
\hline Variable & $\begin{array}{c}\text { MC group } \\
\mathrm{n}=38\end{array}$ & $\begin{array}{l}\text { LC group } \\
n=35\end{array}$ & $p$-Value \\
\hline \multicolumn{4}{|l|}{ Residual abdominal symptoms } \\
\hline Yes/no/could not say & $79.0 \% / 15.6 \% / 5.2 \%$ & $73.3 \% / 17.1 \% / 8.6 \%$ & 0.61 \\
\hline \multicolumn{4}{|l|}{ Right upper quadrant pain attacks } \\
\hline Yes/no/could not say & $5.2 \% / 84.2 \% / 10.5 \%$ & $7.9 \% / 81.6 \% / 10.5 \%$ & 0.89 \\
\hline \multicolumn{4}{|l|}{ Reflux symptoms } \\
\hline Less/not changed/more & $31.6 \% / 63.2 \% / 5.2 \%$ & $26.3 \% / 52,6 \% / 21,1 \%$ & 0.16 \\
\hline \multicolumn{4}{|l|}{ Diarrhea or loose stools } \\
\hline Less/not changed/more & $5.3 \% / 78.9 \% / 15.8 \%$ & $2.6 \% / 68.4 \% / 29.0 \%$ & 0.14 \\
\hline \multicolumn{4}{|l|}{ Avoid fatty/fried food } \\
\hline Yes/no/could not say & $13.2 \% / 84.2 \% / 2.6 \%$ & $18.4 \% / 81.6 \% /-$ & 0.90 \\
\hline \multicolumn{4}{|l|}{ Overall satisfaction with the procedure } \\
\hline Satisfied/dissatisfied/could not say & $91.1 \% / 2.6 \% / 5.3 \%$ & $97.4 \% / 2.6 \% /-$ & 0.40 \\
\hline \multicolumn{4}{|l|}{ Cosmetic satisfaction } \\
\hline Mean NRS (SD) & $8.1(1.7)$ & $8.4(1.8)$ & 0.52 \\
\hline \multicolumn{4}{|l|}{ Recommend the procedure to others } \\
\hline Yes/no/could not say & $81.6 \% / 15.8 \% / 2.6 \%$ & $97.4 \% /-/ 2.6 \%$ & 0.12 \\
\hline \multicolumn{4}{|l|}{ Quality of life } \\
\hline Better/not changed/worse & $92.1 \% / 7.9 \% /-$ & $89.5 \% / 10.5 \% /-$ & 0.60 \\
\hline
\end{tabular}

NRS: Numeric rating scale; SD: standard deviation; MC: minicholecystectomy; LC: laparoscopic cholecystectomy.

Table II. Baseline clinical data of study patients in the minicholecystectomy (MC) and laparoscopic cholecystectomy (LC) patients. Data are mean $(S D)$ or number of cases.

\begin{tabular}{lccc}
\hline Variable & $\begin{array}{c}\text { MC group } \\
\mathrm{n}=44\end{array}$ & $\begin{array}{c}\text { LC group } \\
\mathrm{n}=44\end{array}$ & $p$-Value \\
\hline Age (years) & $44(14)$ & $43(12)$ & 0.74 \\
Gender (male/female) & $8 / 36$ & $3 / 41$ & 0.11 \\
Height $(\mathrm{cm})$ & $167(7)$ & $167(8)$ & 0.95 \\
Weight $(\mathrm{kg})$ & $68(11)$ & $26.1(3.2)$ & 0.07 \\
BMI $\left(\mathrm{kg} / \mathrm{m}^{2}\right)$ & $24.5(3.3)$ & $57(25)$ & 0.034 \\
Operative time (min) & $55(14)$ & $113(22)$ & 0.21 \\
Time at operative theater (min) & $107(16)$ & $29(37)$ & 0.15 \\
Perioperative bleed $(\mathrm{ml})$ & $25(34)$ & $6.6(2.6)$ & 0.59 \\
Length of the skin incisions $(\mathrm{cm})$ & $4.6(1.1)$ & $32 / 12 / 0$ & $<0.001$ \\
ASA $1 / 2 / 3$ & $33 / 10 / 1$ & 2 & 0.95 \\
Conversions & 1 & & \\
\hline
\end{tabular}

BMI: Body mass index; ASA: American Society of Anaesthesiologists Physical Status Score.

Table III. The results of RAND-36 at 8 years.

\begin{tabular}{lcccc}
\hline RAND-36 & MC and LC combined & MC group & LC group & $p$-Value \\
\hline Physical functioning & $89.9(16.4)$ & $93.1(9.2)$ & $86.6(21.2)$ & 0.168 \\
Social functioning & $88.7(18.0)$ & $94.6(12.0)$ & $82.7(21.1)$ & 0.019 \\
Vitality & $69.0(21.7)$ & $72.5(20.4)$ & $65.2(23.0)$ & 0.240 \\
Mental health & $77.0(17.4)$ & $79.2(15.5)$ & $74.7(19.3)$ & 0.360 \\
Role physical & $89.9(16.4)$ & $95.0(20.4)$ & $76.0(40.5)$ & 0.043 \\
Role emotional & $85.5(33.2)$ & $98.7(6.7)$ & $74.7(41.1)$ & 0.008 \\
Bodily pain & $80.0(23.3)$ & $83.3(20.1)$ & $76.6(26.3)$ & 0.312 \\
General health & $71.9(21.7)$ & $74.8(17.3)$ & $68.8(25.4)$ & 0.327 \\
\hline
\end{tabular}

MC: Minicholecystectomy; LC: laparoscopic cholecystectomy. Values are means (standard deviation). 


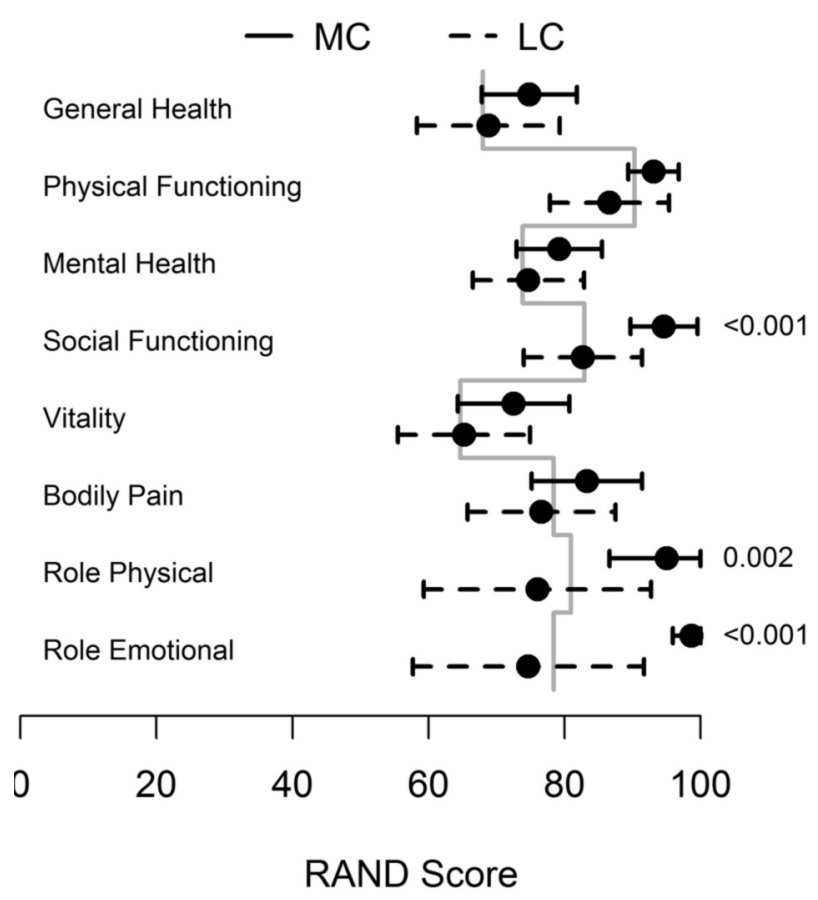

Figure 2. The 8-year postoperative RAND-36 counts in the minicholecystectomy $(M C)$ and laparoscopic cholecystectomy $(L C)$ groups versus the age- and gender-adjusted Finnish reference scores (dashed line) (9).

survey during the 3-year study period and the overall $p$-values were statistically significant in vitality, mental health, role physical and bodily pain domains (11). However, at mid-term, no significant differences in residual symptoms, de novo symptoms, QoL or cosmesis measures between MC and LC patients were shown. In the current article, the long-term RAND-36 showed three domains with significantly improved health related QoL scores in MC versus LC patients.

While considering the strengths of the present study, we feel that language bias might not exist, since the Finnish version of the RAND-36 includes only a few minor translation modifications, which were made in the translation process and wording of some items to make them more appropriate to the Finnish culture (9). We acknowledge that there are some limitations of this study. Firstly, this study lacks preoperative and earlier follow up RAND-36 interviews in this patient cohort. Secondly, due to lack of previous follow-up data, linear mixed effect model analysis was not possible in this study cohort. It is to be admitted that the small number of patients may have affected our final results, however, this could be taken in consideration when planning future studies.

We have previously applied a structured telephone-interview to assess long-term residual and de novo symptoms, QoL and cosmesis between MC and LC patients $(10,11)$. This structured interview takes 15 minutes to register residual abdominal symptoms, right upper quadrant pain, reflux symptoms, diarrhea/loose stools, fatty/fried food, overall satisfaction with the procedure, cosmetic satisfaction, recommend the procedure to others and QoL. We found no statistically significant health status differences using a structured telephone-interview in comparing the MC and LC groups.

\section{Conclusion}

The results of our study support the reliability of the Finnish version of the RAND-36 as a measure of QoL following cholecystectomy.

\section{Conflicts of Interest}

The Authors have no conflicts of interest or financial ties to disclose regarding this study.

\section{Authors' Contributions}

All Authors contributed to the collection and analysis of data, drafting and revising the manuscript, read and approved the final manuscript.

\section{Acknowledgements}

The study was funded by the Finnish Cultural Foundation (PohjoisSavon rahasto) and the Päivikki and Sakari Sohlberg Foundation.

\section{References}

1 Pallis AG and Mouzas IA: Instruments for quality of life assessment in patients with gastrointestinal cancer. Anticancer Res 24: 2117-2121, 2004. PMID: 15274411.

2 Marchiori D, Bertaccini A, Manferrari F, Ferri C and Martorana G: Pelvic floor rehabilitation for continence recovery after radical prostatectomy: role of a personal training re-educational program. Anticancer Res 30: 553-556, 2010. PMID: 20332469.

3 Pilger A, Richter R, Fotopoulou C, Beteta C, Klapp C and Sehouli J: Quality of life and sexuality of patients after treatment for gynaecological malignancies: results of a prospective study in 55 patients. Anticancer Res 32: 5045-5049, 2012. PMID: 23155277.

4 Eskelinen M, Korhonen R, Selander T and Ollonen P: Beck depression inventory as a predictor of long-term outcome among patients admitted to the breast cancer diagnosis unit: A 25-year cohort study in Finland. Anticancer Res 37: 819-824, 2017. PMID: 28179336. DOI: 10.21873/anticanres.11383

5 Eskelinen M, Selander T, Ollonen $\mathrm{P}$ and Korhonen R: Moderate/severe depression (MADRS) can affect the quality of life and outcome among patients admitted to breast cancer diagnosis unit. Anticancer Res 37: 2641-2647, 2017. PMID: 28476839. DOI: 10.21873/anticanres.11611

6 Eskelinen M and Ollonen P: Assessment of general anxiety in patients with breast disease and breast cancer using the Spielberger STAI self-evaluation test: A prospective case-control 
study in Finland. Anticancer Res 31: 1801-1806. 2011. PMID: 21617243.

7 Saimanen I, Kärkkäinen J, Selander T, Purdy M, Kokki M, Kokki $\mathrm{H}$ and Eskelinen M: Plasma catalase in relation to pain following midline laparotomy: A prospective study of patients with benign diseases and patients with cancer. Anticancer Res 38: 6479-6484, 2018. PMID: 30396975. DOI: 10.21873/ anticanres.13011

8 Kuosmanen V, Ruottinen M, Rahkola D, Saimanen I, Kaaronen V, Selander T, Purdy M, Kokki H, Kokki M and Eskelinen M: Brief pain inventory (BPI) health survey after midline laparotomy with the rectus sheath block (RSB) analgesia: A randomised trial of patients with cancer and benign disease. Anticancer Res 39: 6751-6757, 2019. PMID: 31810940. DOI: 10.21873/anticanres. 13890

9 Aalto A-M, Aro AR and Teperi J: Rand-36 as a measure of health-related quality of life. Reliability, construct validity and reference values in the Finnish general population. Helsinki: National Research and Development Center for Welfare and Health. (Research \#101 In Finnish, summary in English), 1999.

10 Aspinen S, Kärkkäinen J, Harju J, Juvonen P, Kokki H and Eskelinen M: Improvement in the quality of life following cholecystectomy: a randomized multicenter study of health status (RAND-36) in patients with laparoscopic cholecystectomy versus minilaparotomy cholecystectomy. Quality Life Res 26: 665-671, 2017. PMID: 28004321. DOI: 10.1007/s11136-016$1485-1$
11 Saimanen I, Kuosmanen V, Rahkola D, Selander T, Kärkkäinen J, Harju J, Aspinen S and Eskelinen M: RAND-36-Item Health Survey: A comprehensive test for long-term outcome and health status following surgery. Anticancer Res 39: 2927-2933, 2019. PMID: 31177131. DOI: 10.21873/anticanres.13422.

12 Harju J, Juvonen P, Kokki H, Remes V, Scheinin T and Eskelinen M: Minilaparotomy cholecystectomy with ultrasonic dissection versus laparoscopic cholecystectomy with electrosurgical energy: A randomized multicenter study. Scandinavian J Gastroenterol 48: 1317-1323, 2013. PMID: 23971855. DOI: $10.3109 / 00365521.2013 .822545$

Received December 14, 2020

Revised December 30, 2020

Accepted December 31, 2020 